\title{
Central Subclavian Catheterization in Newborns: Single-Center Experience
}

\author{
Selcuk Gurel ${ }^{10} \quad$ Ahmet Gözen ${ }^{2(10}$ Mehmet Selcuk Bektas ${ }^{3(0)}$ \\ 1 Department of Pediatrics, Oztan Hospital, Usak, Turkey \\ ${ }^{2}$ Department of Pediatric Surgery, Lokman Hekim Van Hospital, Van, \\ Turkey \\ Address for correspondence Selcuk Gurel, MD, Kemalöz, Atatürk \\ Boulevard No:139, 64200 Merkez/ Uşak, Turkey \\ (e-mail: gurelselcuk@gmail.com).
}

${ }^{3}$ Department of Pediatrics, Lokman Hekim Van Hospital, Van, Turkey

J Child Sci 2022;12:e5-e8.

\begin{abstract}
Our main aim of this article was to show that central venous catheter (CVC) can be an easyto-use, less-complicated catheter application such as peripherally inserted central catheter and umbilical catheter placement in the neonatal intensive care unit. We here described our experience with subclavian vein catheterization. Neonates who had venous access through subclavian central catheterization were assessed retrospectively. Data such as gestational age, age at the time of catheter insertion, birth weight, and gender were collected. In addition, problems related to catheterization during hospitalization were documented. This study comprised 40 newborns, 22 male and 18 female, with a mean gestational week of $29.57 \pm 3.80$ weeks and a mean gestational weight of $2067.50 \pm 545.97 \mathrm{~g}$. Due to occlusion, catheters were switched in five cases twice and in three cases once, totaling 53 catheterizations on 40 newborns. None of our patients had pneumothorax or hemothorax.

Keywords

- newborn

- CVC

- NICU

- catheterization On the postoperative 8th and 21st days, the catheter was withdrawn due to catheter infection in two (5\%) patients, and catheter cultures revealed coagulase negative Staphylococcus aureus in both cases. Even in preterm infants, subclavian central venous catheterization is a safe and straightforward technique of gaining venous access in expert hands in the neonatal intensive care unit.
\end{abstract}

\section{Introduction}

Compared with peripheral lines, central venous catheters (CVCs) enable more secure venous access for a longer period of time, which is essential for whole parenteral feeding and administration of intravenous fluids and drugs to infants admitted in neonatal intensive care units (NICUs). ${ }^{1,2}$ When an alternate peripheral line cannot be established, umbilical venous catheters, peripherally inserted central catheters (PICCs), and CVCs are the main methods of venous access in neonates. CVCs have become popular, especially in premature neonates, due to their importance and ease of insertion by qualified clinicians. ${ }^{3}$ Furthermore, a recent study found that preterm children with very low birth weights who

received

November 10, 2021

accepted

November 24, 2021
DOI https://doi.org/

10.1055/s-0041-1741425.

ISSN 2474-5871. had central catheters gained considerably more weight and spent less time in the hospital than those who did not. ${ }^{4}$ However, complications such as misplacement, infections, and thrombosis can occur when a CVC is inserted in a severely ill, very small infant. ${ }^{5}$

Umbilical venous catheters are preferred choices in low birth infants, since their indwelling is easy and fast. However, they are associated with increased risk of misplacement, thrombosis, and late onset sepsis especially when they are used for more than 5 to 7 days. ${ }^{6,7}$ Other alternatives for venous access are PICCS that also have the similar infection rates especially among preterm neonates born at $<30$ weeks of gestation. ${ }^{5}$

In adults, subclavian CVCs were reported to have the lowest infection rates. ${ }^{8}$ However, the risks of subclavian (c) 2022. The Author(s).

This is an open access article published by Thieme under the terms of the Creative Commons Attribution License, permitting unrestricted use, distribution, and reproduction so long as the original work is properly cited. (https://creativecommons.org/licenses/by/4.0/)

Georg Thieme Verlag KG, Rüdigerstraße 14, 70469 Stuttgart, Germany 
CVCs, such as pneumothorax and hemothorax, limit their use in newborns, particularly those with low birth weight. ${ }^{8,9}$ Although subclavian catheterization is not difficult in skilled hands, there is little information in the literature for infants in NICUs.

In this research, our main aim is to show that CVC can be an easy-to-use, less-complicated catheter application such as PICC and umbilical catheter placement in the NICU. We aimed to report our experience with subclavian vein catheterization and outcomes of subclavian vein catheterization among infants having low birth weights or low gestational weeks.

\section{Material and Method}

This retrospective study was conducted in Van Lokman Hekim Hospital between October 2014 and October 2016. The study protocol was approved by the local ethics committee.

\section{Patients}

A total of 40 infants who were hospitalized in the NICU between October 2014 and October 2016 were retrospectively investigated. Infants who had subclavian central catheterization for venous access were evaluated. None of the patients who underwent CVC were intubated. Patients were on nasal continuous positive airway pressure or oxygen by Hood during the procedure. These procedures were performed at the incubator or in an open neonatal bed. An alternative peripheral venous line was not present in any of the infants. Data was obtained from the patient records, and any patients with missing information were excluded from the study.

Gestational age, age of babies at the time of catheter indwelling, birth weight, and gender of all infants were recorded. Moreover, complications associated with subclavian central venous catheterization during hospitalization were also recorded.

\section{Catheter Insertion}

The same pediatric surgeon used the Seldinger procedure to place 4fr, 22 G, 8 cm FORNA catheters (ROYAL FORNA Zhuhai, PRC) with double lumens in all patients. ${ }^{10}$

Ketamine and dormicum were administered to patients as sedoanalgesia. After sedoanalgesia, a shoulder roll was placed while the patient was in a supine position. The patient was then placed in Trendelenburg position. The operational region was cleaned and disinfected with $2 \%$ chlorhexidine. The subclavian vein was reached by inserting the needle at a 45-degree angle slightly below the distal third of the clavicle medially. The subclavian catheter was inserted with intermittent negative pressure applications. The guidewire was moved further. When the arrhythmia was defined on the electrocardiogram, the movement of the guidewire was stopped. With the perforator sent over the guidewire, the line for the catheter was provided and then the CVC was sent over the guidewire from this line. After insertion of the catheter, the guidewire was withdrawn. The catheter was fixed on the skin with $3 / 0$ silk and cleaned with heparinized serum physiologic.

\section{Follow-Up}

Chest X-rays were collected on the 1st and 24th hours after surgery. Daily catheter dressings were performed with chlorhexidine. All catheters were removed when the line was no longer required. There was no hemothorax or pneumothorax in any case. However, the catheter was displaced in the opposite subclavian or jugular in some cases.

\section{Statistical Analysis}

The study population's characteristics were determined using descriptive statistics, and continuous variables were reported using means, medians, maximum, and lowest values. SPSS version 21 was used to analyze the data.

\section{Results}

A total of 40 infants, 22 males and 18 females, were included in this study. - Table 1 summarizes the gestational ages, catheter insertion ages, catheter periods, and birth weight of the neonates.

The catheter was inserted on the right side in 36 cases and on the left side in four infants. All complications associated with subclavian central venous catheterization were recorded. The catheter was swapped twice in five cases and once in three cases due to occlusion by the guidewire. So, a total of 53 catheterizations on 40 infants were performed. Pneumothorax or hemothorax was not observed in any patient. On the 8 th and 21st postoperative days, the catheter was withdrawn due to catheter infection in two patients, and catheter cultures revealed coagulase positive Staphylococcus aureus in both cases.

When the newborns' gestational ages were examined, 20 of them were between 24 and 28 weeks gestational age,

Table 1 General characteristics of the study population

\begin{tabular}{|l|l|l|l|l|}
\hline & Median & Minimum & Maximum & Mean \pm SD \\
\hline Gestational week & 29 & 24 & 40 & $29.57 \pm 3,80$ \\
\hline Birth weight $(\mathrm{g})$ & 2,000 & 1,050 & 3,100 & $2067.50 \pm 545.97$ \\
\hline Age during catheter insertion (d) & 14 & 1 & 30 & $12.55 \pm 7.35$ \\
\hline Catheter usage period (d) & 20 & 7 & 52 & $21.37 \pm 9.77$ \\
\hline
\end{tabular}

Abbreviation: SD, standard deviation. 
while the other 20 were born more than 28 weeks gestational age. When the infants were analyzed regarding their birth weights, 7 of them were between 1,050 and $1,500 \mathrm{~g} ; 13$ of them were between 1,500 and 2,000 g; and 20 of them were heavier than $2000 \mathrm{~g}$. Catheters were implanted in 12 of the infants within the first week of their lives, and catheterization was performed in 11 of them during the second week. Catheters were used for less than 10 days in 4 patients, for 10 to 20 days in 18 of them and for more than 20 days in 18 patients.

Mortality was not reported in any of the study participants. All infants were discharged as healthy from the NICU.

\section{Discussion}

In this research, we have reported our experience with subclavian central venous catheterization in neonates admitted to the intensive care unit. A total of 53 successful catheterizations were performed on 40 infants. We had to withdraw the catheters in only two (5\%) patients due to infection and we did not determine any cases with pneumothorax or hemothorax. With these findings, subclavian central venous catheterization would be considered a safe and useful option in the hands of experienced clinicians for infants requiring central catheterization without alternative peripheral venous lines, even in premature babies with low birth weights $(<2,000 \mathrm{~g})$ or gestational ages $(<28$ weeks).

Venous access is critical for newborns who are hospitalized in NICU, for giving both nourishment and drugs. Many times, central venous lines are mandatory in the NICU in premature babies to provide basic requirements for the infants.

The data about the insertion of a subclavian central venous line in the NICU is limited in the literature. In a retrospective study, Lausten-Thomsen et al reported the successful subclavian CVC insertion rate with an ultrasound-guided supraclavicular approach as 97.3\% in 105 newborns weighing less than 5,000 g and hospitalized in the NICU. ${ }^{11}$ Guilbert et al also reported the success rate of supraclavicular ultrasound-guided subclavian vein catheterization as $97.6 \%$, with only one failure in 40 children in ICU. ${ }^{12}$ Araujo et al reported that they were successful in $89.2 \%$ of percutaneous subclavian central venous catheterizations. ${ }^{13}$ Although we did not use an ultrasound guided method, we had a $100 \%$ successful insertion rate with the Seldinger technique.

In their report, García and Torres-Yáñez reported the most frequent complications of central venous catheterizations as colonization and catheter-related bacteremia, determined in $\sim 30 \%$ of 123 newborns. ${ }^{14}$ Sheridan and Weber reported the catheter sepsis rate as $7.4 \%$ in 245 children with an average age of $6.4 \pm 5.1$ years after the insertion of a subclavian catheter without any pneumothorax, vascular lacerations, acute thromboses, or catheter emboli. ${ }^{15}$ Although our patient population comprised neonates in a NICU, the lower infection rates determined in this study may be attributed to the experienced and careful health personnel working in the NICU.
We found S. aureus in blood cultures in both infants who had catheter-associated infections, which is also one of the most commonly defined bacteria in NICU device-associated infections. ${ }^{16}$ Premature birth, low weight, immunodeficiency, prolonged hospital stay, and antibiotic use are some of the risk factors related with S. aureus infections in the NICU. ${ }^{17-19}$

Kaji et al reported the pneumothorax rate as $0.9 \%$ in 230 CVCs inserted into the subclavian vein using the landmark technique in pediatric patients. ${ }^{20}$ In another study, 4 cases of pneumothorax (2\%), 2 cases of hemothorax (1\%), and 1 case of hydrothorax (0.5\%) were documented among 197 subclavian central venous catheterizations in pediatric patients with a median age of 5 months. ${ }^{13}$ In this study, we found no cases of pneumothorax or hemothorax following the procedure.

The optimal time between the insertion and the removal of the central catheters, named as the dwell time, is still not clearly known. ${ }^{21}$ However, the literature data about the association of longer durations and increased risk for bacterial infections is controversial. ${ }^{22,23}$ In this study, the catheters were used for 7 to 52 days, with a mean usage of $21.37 \pm 9.77$ days. Catheter-related infections were reported in two cases on the 8 th and 21 st days, which could not be associated with the time.

Umbilical venous catheters and PICCs are the other alternatives when a peripheral line cannot be established. Umbilical venous catheters are frequently used in neonates since they provide fast and easy access. However, umbilical catheters were reported to have a higher infection rate than nonumbilical central catheters, and longer umbilical catheter dwell time was also increasing the infection rate. ${ }^{24}$ More than 7 days of usage of umbilical venous catheters was reported to increase the catheter-related infection risk, and if required for more than 7 days, replacement of umbilical venous catheters with other central venous access was advised. ${ }^{7}$ However, Zingg et al determined that the highest rates of central line-associated bloodstream infections and clinical sepsis were reported among neonates weighing $750 \mathrm{~g}$ or lower and having PICCs. ${ }^{22}$ PICCs may be preferred in some cases due to their ease of insertion and prolonged usage periods. In a recent study, the infection rate of PICCs was reported as $25.4 \%$ among newborn infants weighing between 500 and $1,499 \mathrm{~g}^{25}$ In another recent study, the rate of catheter-related bloodstream infection was reported as $10.3 \%$ among 39 very low birth weight infants with a mean birth weight of $1042.7 \mathrm{~g}$ and mean gestational age of 28.5 weeks. ${ }^{26}$ Studies comparing those alternatives for the peripheral lines among neonates, especially in terms of complications, are warranted.

There are some limitations to this study that should be mentioned. First is the low number of infants included in this study. Second, we aimed to compare infants with different gestational ages and birth weight regarding the subclavian CVC-associated complications, but since our complication rate was very low, we could not perform a comparison. Moreover, comparing subclavian venous catheters with other methods of venous access may be more efficient, which may be the topic of another study. 


\section{Conclusion}

In our experience, complications such as pneumothorax, vascular lacerations, acute thrombosis, catheter embolism, and especially sepsis are rare with subclavian venous catheterization. Subclavian central venous catheterization is a safe and simple method of gaining venous access in trained hands in the NICU, even in small or preterm infants. Larger studies comparing different venous accesses in NICUs are warranted, especially in terms of complications.

\section{Conflict of Interest}

None declared.

\section{References}

1 Crowley JJ. Vascular access. Tech Vasc Interv Radiol 2003;6(04): 176-181

2 Janes M, Kalyn A, Pinelli J, Paes B. A randomized trial comparing peripherally inserted central venous catheters and peripheral intravenous catheters in infants with very low birth weight. J Pediatr Surg 2000;35(07):1040-1044

3 Stolfi I, Boccanera F, Chiara C, et al. Central venous lines and how to manage them. Early Hum Dev 2009;85(Suppl 10):S83-S84

4 Yang ZM, Wang SN, Ma YL, Yang XL, Zhang WY, Zhu MY. [Application of umbilical venous catheter combined with peripherally inserted central catheter in very low birth weight infants]. Zhongguo Dang Dai Er Ke Za Zhi 2013;15(05):353-355

5 Shalabi M, Adel M, Yoon E, Aziz K, Lee S, Shah PSCanadian Neonatal Network. Risk of infection using peripherally inserted central and umbilical catheters in preterm neonates. Pediatrics 2015;136(06):1073-1079

$6 \mathrm{Kim}$ JH, Lee YS, Kim SH, Lee SK, Lim MK, Kim HS. Does umbilical vein catheterization lead to portal venous thrombosis? Prospective US evaluation in 100 neonates. Radiology 2001;219(03):645-650

7 Butler-O'Hara M, D'Angio CT, Hoey H, Stevens TP. An evidencebased catheter bundle alters central venous catheter strategy in newborn infants. J Pediatr 2012;160(06):972-7.e2

8 Youn SH, Lee JC, Kim Y, Moon J, Choi Y, Jung K. Central venous catheter-related infection in severe trauma patients. World J Surg 2015;39(10):2400-2406

9 Finck C, Smith S, Jackson R, Wagner C. Percutaneous subclavian central venous catheterization in children younger than one year of age. Am Surg 2002;68(04):401-404

10 Seldinger SI. Catheter replacement of the needle in percutaneous arteriography; a new technique. Acta Radiol 1953;39(05): 368-376

11 Lausten-Thomsen U, Merchaoui Z, Dubois C, et al. Ultrasoundguided subclavian vein cannulation in low birth weight neonates. Pediatr Crit Care Med 2017;18(02):172-175

12 Guilbert AS, Xavier L, Ammouche C, et al. Supraclavicular ultrasound-guided catheterization of the subclavian vein in pediatric and neonatal ICUs: a feasibility study. Pediatr Crit Care Med 2013; 14(04):351-355

13 Araujo CC, Lima MC, Falbo GH. Percutaneous subclavian central venous catheterization in children and adolescents: success, complications and related factors. J Pediatr (Rio J) 2007;83(01): 64-70

14 García HJ, Torres-Yáñez HL. [Survival and complication rate of central venous catheters in newborns]. Rev Med Inst Mex Seguro Soc 2015;53(Suppl 3):S300-S309

15 Sheridan RL, Weber JM. Mechanical and infectious complications of central venous cannulation in children: lessons learned from a 10-year experience placing more than 1000 catheters. J Burn Care Res 2006;27(05):713-718

16 Urzedo JE, Levenhagen MM, Pedroso RS, Abdallah VO, Sabino SS Brito DV. Nosocomial infections in a neonatal intensive care unit during 16 years: 1997-2012. Rev Soc Bras Med Trop 2014;47(03): $321-326$

17 Escobar GJ, Li DK, Armstrong MA, et al. Neonatal sepsis workups in infants $>/=2000$ grams at birth: a population-based study. Pediatrics 2000;106(2 Pt 1):256-263

18 de Almeida Silva H, Steffen Abdallah VO, Carneiro CL, Gontijo PP PP. Infection and colonization by Staphylococcus aureus in a high risk nursery of a Brazilian teaching hospital. Braz J Infect Dis 2003; 7(06):381-386

19 Aslam N, Mehdi N, Izhar M. Root cause analysis of methicillin resistant Staphylococcus aureus bacteraemia. J Pak Med Assoc 2015;65(10):1089-1093

20 Kaji T, Kawano T, Yamada W, et al. The changing profile of safe techniques for the insertion of a central venous catheter in pediatric patients - improvement in the outcome with the experiences of 500 insertions in a single institution. J Pediatr Surg 2016;51(12):2044-2047

21 Greenberg RG, Cochran KM, Smith PB, et al. Effect of catheter dwell time on risk of central line-associated bloodstream infection in infants. Pediatrics 2015;136(06):1080-1086

22 Zingg W, Posfay-Barbe KM, Pfister RE, Touveneau S, Pittet D. Individualized catheter surveillance among neonates: a prospective, 8-year, single-center experience. Infect Control Hosp Epidemiol 2011;32(01):42-49

23 Smith PB, Benjamin DK Jr, Cotten CM, et al. Is an increased dwell time of a peripherally inserted catheter associated with an increased risk of bloodstream infection in infants? Infect Control Hosp Epidemiol 2008;29(08):749-753

24 Yumani DF, van den Dungen FA, van Weissenbruch MM. Incidence and risk factors for catheter-associated bloodstream infections in neonatal intensive care. Acta Paediatr 2013;102(07):e293-e298

25 Rangel UV, Gomes Junior SC, Costa AM, Moreira ME. Variables associated with peripherally inserted central catheter related infection in high risk newborn infants. Rev Lat Am Enfermagem 2014;22(05):842-847

26 Cheong SM, Totsu S, Nakanishi H, Uchiyama A, Kusuda S. Outcomes of peripherally inserted double lumen central catheter in very low birth weight infants. J Neonatal Perinatal Med 2016;9 (01):99-105 